\title{
RING ELEMENTS AS SUMS OF UNITS
}

\author{
CHARLES LANSKI AND ATTILA MARÓTI
}

\begin{abstract}
In an Artinian ring $R$ every element of $R$ can be expressed as the sum of two units if and only if $R / J(R)$ does not contain a summand isomorphic to the field with two elements. This result is used to describe those finite rings $R$ for which $\Gamma(R)$ contains a Hamiltonian cycle where $\Gamma(R)$ is the (simple) graph defined on the elements of $R$ with an edge between vertices $r$ and $s$ if and only if $r-s$ is invertible. It is also shown that for an Artinian ring $R$ the number of connected components of the graph $\Gamma(R)$ is a power of 2 .
\end{abstract}

\section{INTRODUCTION}

Unless otherwise stated it is assumed that every ring in this paper has a multiplicative identity. Let $J(R)$ denote the Jacobson radical of a ring $R$ and let $G F(2)$ denote the field of two elements.

Theorem 1. Let $R$ be an Artinian ring. Then every element of $R$ can be expressed as the sum of two units if and only if $R / J(R)$ does not contain a summand isomorphic to $G F(2)$.

By Theorem 1, one may also construct non-Artinian rings $R$ with the property that every element of $R$ can be expressed as the sum of two units. In general, one may take $R=F[X] / I$ where $F$ is an Artinian ring so that $F / J(F)$ does not contain a summand isomorphic to $G F(2), F[X]$ is a polynomial ring over $F$ where $X$ is an infinite set of commuting indeterminates, and $I$ is the ideal in $F[X]$ generated by the squares of the indeterminates. Indeed, every element $r$ of $R$ has the form $r=f+h(X)$ where $f \in F$ and $h(X)$ is nilpotent. By the choice of $F$, there exist units $f_{1}, f_{2}$ with $f=f_{1}+f_{2}$ and so $r=f_{1}+\left(f_{2}+h(X)\right)$ expressed as the sum of two units.

For a ring $R$ let $\Gamma(R)$ be the graph defined on the elements of $R$ with an edge between vertices $r$ and $s$ if and only if $r-s$ is invertible. This graph was defined in a paper of Lucchini and Maróti [4] where it was shown that $\Gamma(R)$ is arc transitive (vertex-transitive and edge-transitive)(see the beginning of Section 7 of [4]), and also that for an Artinian ring $R$, the chromatic number of $\Gamma(R)$ is equal to the maximal cardinality of a complete subgraph in $\Gamma(R)$ (see Theorem 6.1 of [4]). (The chromatic number of a (simple) graph is the least cardinality of colors needed to color the vertices of the graph in such a way that the endpoints of every edge receive different colors.)

Let $R$ be an Artinian ring with the property that $R / J(R)$ does not contain a summand isomorphic to $G F(2)$. We claim that the graph $\Gamma(R)$ is connected and has diameter at most 2. By Theorem 1 we know that every element of $R$ can be expressed as a sum of two units, so for any different $a, b \in R$ there are units $u$ and $v$ with $a-b=u+v$. Thus $b$ is connected to $b+u$, and this vertex is connected to $b+u+v=a$. This proves that every vertex $x$ is connected with every vertex different from $x$ by a path of length at most 2 .

\footnotetext{
${ }^{1}$ The research of the second author was supported by a Marie Curie International Reintegration Grant within the 7th European Community Framework Programme and partially by grants OTKA T049841 and OTKA NK72523.

Date: 10th April, 2009.
} 
The following theorem shows that $\Gamma(R)$ can have more than one connected component for an Artinian ring $R$; in fact, the number of connected components in $\Gamma(R)$ for an Artinian ring $R$ is always a power of 2. A bipartite graph is a graph whose vertices can be divided into two disjoint sets $U$ and $V$ such that every edge connects a vertex in $U$ to one in $V$; that is, $U$ and $V$ are independent sets ( $U$ and $V$ induce empty subgraphs).

Theorem 2. Let $R$ be an Artinian ring with the property that $R / J(R)$ has $k$ summands isomorphic to $G F(2)$ for some positive integer $k$.

(1) Then $\Gamma(R)$ contains $2^{k-1}$ connected components each of which is a bipartite graph.

(2) There exist mutually orthogonal idempotents $e_{1}, \ldots, e_{k}$ in $R$ such that for every element $r$ in $R$ there exist $\epsilon_{1}, \ldots, \epsilon_{k} \in\{0,1\}$ and units $u$ and $v$ in $R$ so that $r=\epsilon_{1} e_{1}+\ldots+\epsilon_{k} e_{k}+u+v$.

For a non-Artinian ring $R$ the graph $\Gamma(R)$ may contain infinitely many connected components each of which is a complete subgraph. Indeed, consider the polynomial ring $R=D[x]$ in one variable over a division ring $D$.

In general, for any ring $R$, the connected components of $\Gamma(R)$ are all isomorphic to $\Gamma(S)$ where $S$ is the subring of $R$ generated by the group of units of $R$. Indeed, if $S$ denotes the subring of $R$ generated by the group of units of $R$, then, for each $r \in R$, the set $r+S$ is the set of vertices of the connected component $\Gamma_{r}$ of $\Gamma(R)$ containing $r$. Clearly, $\Gamma_{0} \cong \Gamma(S)$ and also $\Gamma_{0} \cong \Gamma_{r}$ via the restriction of the automorphism of $\Gamma(R)$ sending a vertex $x$ to $x+r$.

Theorems 1 and 2 lead to a full description of those finite rings $R$ for which $\Gamma(R)$ contains a Hamiltonian cycle.

Corollary 1. Let $R$ be a finite ring with at least three elements. Then the graph $\Gamma(R)$ contains a Hamiltonian cycle if and only if $R / J(R)$ contains at most one summand isomorphic to $G F(2)$.

The proofs of this note also show that for a finite ring $R$ with at least three elements $\Gamma(R)$ contains a Hamiltonian cycle if and only if every element of $R$ can be expressed as the sum of two or three units.

For a non-empty proper subset $X$ of a finite $\operatorname{ring} R$ let $P_{X}$ be the probability that $r-s$ is a unit where $r$ is chosen uniformly at random from $X$ and $s$ is chosen uniformly at random from $R \backslash X$. Our last result is

Corollary 2. Let $R$ be a finite ring. Then either $P_{X}=0$ for some non-empty proper subset $X$ of $R$, or $1 /(3|R|)<P_{X}$ for every non-empty proper subset $X$ of $R$.

\section{Proof of Theorem 1}

If $U(R)$ denotes the group of units of the ring $R$, then the direct product $U(R) \times$ $U(R)$ acts on $R$ in a natural way. (An element $(u, v)$ of $U(R) \times U(R)$ takes $x \in R$ to $u^{-1} x v$.) For a positive integer $n$ let $M_{n}(D)$ be the full matrix ring consisting of $n \times n$ matrices with entries from the division ring $D$.

Lemma 1. Let $n \geq 2$ and let $D$ be a division ring. Then every $U\left(M_{n}(D)\right) \times$ $U\left(M_{n}(D)\right)$-orbit of $M_{n}(D)$ has a representative whose entries in the main diagonal are 0 's.

Proof. Let $X$ be an arbitrary matrix in $M_{n}(D)$. Let $r$ be its rank. By invertible row and column operations it is possible to eliminate all entries of $X$ apart from the first $r$ entries along the main diagonal. Finally we may multiply the resulting matrix from the right by a cyclic permutation matrix to obtain a matrix all of whose entries on the main diagonal are 0 . 
From Lemma 1 it follows that for $n \geq 2$ and $D$ a division ring, every element of $M_{n}(D)$ can be expressed as the sum of two units. Indeed, let $X \in M_{n}(D)$ be an arbitrary matrix. We know that there exist invertible matrices $U$ and $V$ so that $U^{-1} X V$ has all 0 's on its main diagonal. If $d \in D$ is not zero, then $U^{-1} X V$ is the sum of an upper triangular matrix $A$ with all $d$ 's on the main diagonal and a lower triangular matrix $B$ with all $-d$ 's on the main diagonal. Finally, $X=U A V^{-1}+U B V^{-1}$.

Before we further generalize (see Lemma 2) this latter observation, let us state a well-known fact that is a standard exercise and will be used in several places of this note.

Fact 1. An element of an Artinian ring is invertible if and only if it is invertible modulo the Jacobson radical.

Lemma 2. Let $R$ be an Artinian ring with the property that no summand of $R / J(R)$ is isomorphic to $G F(2)$. Then every element of $R$ is the sum of two units.

Proof. First let $R$ be a division ring $D$ different from $G F(2)$. Then $0=a+(-a)$ for some units $a$ and $-a$, and for every non-zero element $x$ of $D$ we have $x=a+(x-a)$ for some units $a$ and $x-a$. By the observation after Lemma 1, we already know that every element of $R=M_{n}(D)$ is the sum of two units for $n \geq 2$ and $D$ an arbitrary division ring.

If $S$ and $T$ are rings in which every element can be expressed as the sum of two units, then the ring $S \oplus T$ also has this property. Hence, if $R$ is a semi-simple ring with no summand isomorphic to $G F(2)$, then every element of $R$ is the sum of exactly two units.

Finally, to prove the lemma, suppose that $R$ is an Artinian ring with the property that $J(R) \neq 0$ and that no factor of $R / J(R)$ is isomorphic to $G F(2)$. Let $x$ be an arbitrary element of $R$. Then there exist units $a+J(R)$ and $b+J(R)$ in $R / J(R)$ so that $x+J(R)=(a+J(R))+(b+J(R))$. Hence $x=(a+j)+(b+k)$ for some $j, k$ in $J(R)$. But both $a+j$ and $b+k$ are units by Fact 1 .

Lemma 2 provides one implication of Theorem 1. For the other implication let $R$ be an Artinian ring with the property that at least one summand of $R / J(R)$ is isomorphic to $G F(2)$. Let $\varphi_{1}$ be a (natural) projection of $R$ onto one of the $G F(2)$ summands of $R / J(R)$. Then no element of $\varphi_{1}^{-1}(1)$ can be expressed as the sum of two units. This completes the proof of Theorem 1 .

\section{Proof of Theorem 2}

For an arbitrary ring $R$ the graph $\Gamma(R)$ is isomorphic to the Cayley graph $\Gamma(R, U(R))$ of the Abelian group $(R,+)$ with respect to the set $U(R)$. Cayley graphs of groups have been much investigated.

Starting from the set of graphs $\Gamma\left(M_{n}(D)\right)$ of full $n$-by- $n$ matrix algebras $M_{n}(D)$ over division rings $D$ all graphs $\Gamma(R)$ for $R$ an Artinian ring can be built up using two kinds of graph constructions: "blowing up of vertices" and direct products. This observation is based on the Artin-Wedderburn Theorem.

The first construction is related to Fact 1 . Indeed, a consequence of Fact 1 is that $\Gamma(R)$ can be obtained from $\Gamma(R / J(R))$ just by replacing each vertex of $\Gamma(R / J(R))$ by an empty graph of cardinality $|J(R)|$ and by replacing each edge of $\Gamma(R / J(R))$ by a cardinality of $|J(R)|^{2}$ edges running between the relevant two empty graphs replacing the relevant two vertices of $\Gamma(R / J(R)$ ). (Also note that there is no isolated vertex in $\Gamma(R / J(R))$ and hence neither in $\Gamma(R)$.)

The direct product of two (simple) graphs $\Gamma_{1}$ and $\Gamma_{2}$ is the (simple) graph $\Gamma_{1} \times \Gamma_{2}$ whose vertex set is the cartesian product of the vertex sets of $\Gamma_{1}$ and $\Gamma_{2}$, 
and two pairs in $\Gamma_{1} \times \Gamma_{2}$ are connected by an edge if both of their components are connected in $\Gamma_{1}$ and $\Gamma_{2}$. It is easy to see that for arbitrary rings $S$ and $T$ we have $\Gamma(S \oplus T) \cong \Gamma(S) \times \Gamma(T)$.

Now we turn to the proof of part (1) of Theorem 2.

Let $R$ be an Artinian ring with the property that $R / J(R)$ has $k$ summands isomorphic to $G F(2)$ for some positive integer $k$. We must show that $\Gamma(R)$ contains $2^{k-1}$ connected components each of which is a bipartite graph.

By the above, we may assume that $J(R)=0$, that is, $R$ is a direct sum of full matrix algebras over division rings.

Let $S$ be the ring which is the direct sum of $k$ copies of $G F(2)$ and let $T$ be the subring of $R$ which is the direct sum of all but the $k$ copies of $G F(2)$. Then $R=S \oplus T$.

If $T=0$, then $\Gamma(R)$ is isomorphic to the direct product of $k$ copies of $\Gamma(G F(2))$ which is a graph $\Gamma(k)$ on $2^{k}$ vertices and $2^{k-1}$ independent edges. Hence we may assume that $T \neq 0$.

By the above, it is sufficient to show that the graph $\Gamma(k) \times \Gamma(T)$ contains $2^{k-1}$ connected components each of which is a bipartite graph. But this follows from the fact (see Theorem 1) that $\Gamma(T)$ is a connected graph with the property that for any pair of distinct vertices $x$ and $y$ there is a third vertex $z$ which is connected to both $x$ and $y$.

Now we turn to the proof of part (2) of Theorem 2.

Let $R$ be an Artinian ring with the property that $R / J(R)$ has $k$ summands isomorphic to $G F(2)$ for some positive integer $k$. Write $R / J(R)$ in the form $T \oplus S$ where $T$ is the sum of all summands of $R / J(R)$ which are different from $G F(2)$ and $S$ is the sum of all $k$ summands of $R / J(R)$ isomorphic to $G F(2)$. Let $\varphi$ be the composition of the natural projections from $R$ onto $R / J(R)$ and $R / J(R)$ onto $S$. The ring $S$ has $k$ mutually orthogonal idempotents $f_{1}, \ldots, f_{k}$ each of which generates one of the $k$ summands in $S$ isomorphic to $G F(2)$ and $f_{1}+\ldots+f_{k}=1_{S}$. By Proposition 4 on page 54 of [2], there exist mutually orthogonal idempotents $e_{1}, \ldots, e_{k}$ of $R$ with the property that $e_{i} \in \varphi^{-1}\left(f_{i}\right)$ and $2 e_{i} \in J(R)$ for all $i$ with $1 \leq i \leq k$. Let $r$ be an arbitrary element of $R$. Then there exist $\epsilon_{1}, \ldots, \epsilon_{k} \in\{0,1\}$ with the property that $\varphi\left(r-\left(\epsilon_{1} e_{1}+\ldots+\epsilon_{k} e_{k}\right)\right)=0$. But then, by Theorem 1 , there exist units $u^{\prime}, v^{\prime}$ in $T$ and $j_{1}, j_{2} \in J(R)$ so that $r-\left(\epsilon_{1} e_{1}+\ldots+\epsilon_{k} e_{k}\right)=$ $\left(u^{\prime}+1_{S}+j_{1}\right)+\left(v^{\prime}+1_{S}+j_{2}\right)$. Since $u=u^{\prime}+1_{S}+j_{1}$ and $v=v^{\prime}+1_{S}+j_{2}$ are units in $R$ by Fact 1 , this proves part (2) of Theorem 2 .

\section{Proof of Corollary 1}

Corollary 1 and the remark after the statement of Corollary 1 are consequences of Lemmas 3 and 4.

Lemma 3. Let $R$ be an Artinian ring. Then the following three conditions are equivalent.

(1) The factor ring $R / J(R)$ has at most one summand isomorphic to $G F(2)$.

(2) The graph $\Gamma(R)$ is connected.

(3) Every element of $R$ is a sum of two or three units.

Proof. The implication (2) $\Longrightarrow$ (1) follows from part (1) of Theorem 2. Again by part (1) of Theorem 2, for the implication $(1) \Longrightarrow(2)$, it can be assumed that the factor ring $R / J(R)$ has no summand isomorphic to $G F(2)$. But then Theorem 1 implies that $\Gamma(R)$ has diameter at most 2 (since $\Gamma(R)$ is vertex-transitive) hence is connected. Since $\Gamma(R)$ is vertex-transitive, (3) implies that $\Gamma(R)$ has diameter at most 3 and so is connected. To finish the proof of Lemma 3 it is sufficient to show the implication $(1) \Longrightarrow(3)$. By Theorem 1 , it is sufficient to show that if $R$ is 
an Artinian ring with $R / J(R)$ having exactly one summand isomorphic to $G F(2)$, then every element of $R$ is a sum of two or three units. As before, let $\varphi_{1}$ be a (natural) projection of $R$ onto one of the $G F(2)$ summands of $R / J(R)$. Then, by Lemma 2, every element of $\varphi_{1}^{-1}(0)$ is a sum of two units and every element of $\varphi_{1}^{-1}(1)$ is a sum of three units.

Lemma 4. For a finite ring $R$ with at least three elements $\Gamma(R)$ contains a Hamiltonian cycle if and only if $\Gamma(R)$ is connected.

Proof. It is sufficient to show that if $\Gamma(R)$ is connected then $\Gamma(R)$ contains a Hamiltonian cycle for $|R| \geq 3$. So suppose that $\Gamma(R)$ is connected and that $|R| \geq 3$. Notice that $\operatorname{Aut}(\Gamma(R))$ contains a regular Abelian subgroup, namely the additive group $R$, and so we may conclude that the graph $\Gamma(R)$ contains a Hamiltonian cycle by Exercise 12.17 of [3].

\section{Proof of Corollary 2}

The edge expansion $h(G)$ of a graph $G$ on $n$ vertices is defined as

$$
h(G)=\min _{1 \leq|X| \leq n / 2} \frac{|\partial(X)|}{|X|}
$$

where the minimum is over all non-empty sets $X$ of at most $n / 2$ vertices, and $\partial(X)$ stands for the set of edges with exactly one endpoint in $X$.

In the solution of Exercise 11.32 (b) of [3] it is shown that if $G$ is a connected vertex-transitive graph on $n$ vertices with diameter $D$, then for every non-empty subset $X$ of the vertex-set of $G$ of size at most $n / 2$ we have

Hence $h(G) \geq 1 /(2 D)$.

$$
|\partial(X)| /|X| \geq \frac{n-|X|}{D(n-1)} .
$$

Let $G=\Gamma(R)$ for a finite ring $R$. If $\Gamma(R)$ is not connected then taking $X$ to be the vertex-set of a connected component of $\Gamma(R)$ we have

$$
P_{X}=\frac{|\partial(X)|}{|X|(|R|-|X|)}=0
$$

since $\partial(X)=\emptyset$. Otherwise, if $\Gamma(R)$ is connected, then, by Lemma 3, the graph $\Gamma(R)$ has diameter at most 3 , we have seen that $\Gamma(R)$ is vertex-transitive, and so, by the above, $P_{X}>1 /(3|R|)$.

\section{Acknowledgement.}

Since this paper was published Peter Vámos informed us that the topic of ring elements as sums of units had been investigated earlier [1], [5]. Our Theorem 1.1 is a special case of Lemma 2(d) in [5] which is also quoted as (F3) together with (F8) in [1]. The proofs of Theorem 1.1 and [5, Lemma 2(d)] are equal in length. They use a special case of [6, Theorem], which is our observation made after Lemma 2.1. We believe that the graph $\Gamma(R)$ had not been investigated earlier therefore the rest of the paper, our Theorem 1.2, Corollary 1.1, and Corollary 1.2 are unaffected.

\section{REFERENCES}

[1] Ashrafi, N.; Vámos, P. On the unit sum number of some rings. Q. J. Math. 56 (2005), no. 1, $1-12$.

[2] Jacobson, N. Structure of rings. American Mathematical Society Colloquium Publications, Vol. 37, 1956.

[3] Lovász, L. Combinatorial problems and exercises. North-Holland, Amsterdam, 1979.

[4] Lucchini, A.; Maróti, A. Some results and questions related to the generating graph of a finite group. To appear in the Proceedings of the Ischia Group Theory Conference 2008. 
[5] Vámos, P. 2-good rings. Q. J. Math. 56 (2005), no. 3, 417-430.

[6] Zelinsky, D. Every linear transformation is a sum of nonsingular ones. Proc. Amer. Math. Soc. 5, (1954). 627-630.

Charles Lanski, Department of Mathematics, University of Southern California, Los Angeles, CA 90089-2532, USA

E-mail address: clanski@usc.edu

Attila Maróti, MTA Alfréd Rényi Institute of Mathematics, Budapest, Hungary. E-mail address: maroti@renyi.hu 